\title{
Característica de Euler-Poincaré para poliedros $n$-tóricos
}

\author{
Luciene Parron Gimenes Arantes
}

\author{
Wellen Eder Pedrochi ${ }^{1}$
}

Rodrigo Martins

\section{Resumo}

A Característica de Euler-Poincaré é um invariante topológico bem conhecido e o cálculo para superfícies compactas, em geral, depende de uma instrumentação topológica bastante refinada. Entretanto, no caso de poliedros convexos esse invariante é igual a 2 e resulta de uma simples verificação de uma fórmula a qual relaciona o número de vértices $V$, o número de arestas $A$ e o número de faces $F$ de um poliedro, mais especificamente, $V-A+F=2$ (Fórmula de Euler) e isso se dá porque tais poliedros convexos são homeomorfos à esfera. Neste trabalho apresentamos uma demonstração, usando apenas indução finita, para o cálculo desse invariante para uma classe de poliedros não convexos, apresentados de maneira específica, aqui chamados poliedros $n$-tóricos, que são homeomorfos a $n$-toros.

Palavras-chave: Característica de Euler-Poincaré; poliedro não convexo; $n$-toros; poliedros $n$-tóricos

\section{Abstract}

The Euler-Poincaré Characteristic is a well-known topological invariant. The computation for compact surfaces depends on a highly refined topological tools, however in the case of convex polyhedra this invariant is constant equal to two and it follows, because convex polyhedra are homeomorphic to a sphere, from a simple verification of the formula which relates the number of vertices $V$, the number of edges $A$ and the number of faces $F$ of a polyhedron, more precisely, $V-A+F=2$ (Euler's Formula). In this work we present a proof, using only finite induction, for the computation this invariant taking into account, however, only a class of non-convex polyhedra, presented in a specific way, here called $n$-toric polyhedra, which are homeomorphic to an $n$-torus.

Keywords: Euler-Poincaré characteristic; non-convex polyhedra; $n$-torus; $n$-torics polyhedra.

\section{Introdução}

A Fórmula de Euler é um tema de muita relevância e tem sido ensinado nas disciplinas que envolvem Geometria Espacial no Ensino Médio, por exemplo; veja SOUZA(2013) e LIMA(1999). É um resultado muito simples e com um enunciado de fácil compreensão o qual relaciona o número

\footnotetext{
${ }^{1}$ Apoio financeiro Capes
} 
de vértices $V$, o número de arestas $A$ e o número de faces $F$ de um poliedro, mais especificamente, $V-A+F=2$, o que é de fácil ilustração e entendimento.

Poincaré foi o primeiro matemático a compreender que a Fórmula de Euler é um resultado de Topologia, e não de Geometria como se pensava, assim a relação $X(P)=V-A+F$ ficou conhecida como Característica de Euler-Poincaré de um poliedro $P$. Também esse tema foi amplamente discutido em livros, veja por exemplo, LIMA(1991) e LIMA(1999) e nas dissertações GISOLDI(2013) e PEDROCHI(2016).

Segundo E. L. Lima, em LIMA(1991), a demonstração mais divulgada da Fórmula de Euler foi apresentada por Cauchy, depois reproduzida por autores conceituados, como Hilbert-Cohn Vossen e Courant-Robbins numa forma aparentemente compatível com o nível de conhecimento adquirido pelos alunos no Ensino Médio. Nesse mesmo artigo, o próprio Professor E. L. Lima apresentou uma leitura crítica da demonstração de Cauchy verificando que se pode expandir as noções apresentadas, na versão envolvendo poliedros convexos, para poliedros não convexos.

De igual forma, queremos aqui estudar não apenas a Fórmula de Euler, mas a Característica de Euler-Poincaré, da maneira mais simples e informal possível para alcançar o mesmo público apto a compreender essa fórmula para poliedros regulares levando em consideração um conjunto mais amplo de poliedros. Para isso, queremos lançar mão apenas das ferramentas mais elementares de geometria, entre elas a observação construtiva e a recursividade já utilizadas desde os tempos remotos.

\section{A Característica de Euler-Poincaré}

\subsection{Poliedros não convexos}

Foi graças a Poincaré, em 1893, a solução definitiva para a Fórmula de Euler. Poincaré mostrou que dado um poliedro $P$, o número $X(P)=V-A+F$, chamado de Característica de Euler-Poincaré, é um invariante topológico; isto é, dados os poliedros $P$ e $Q$, com mesma Característica de EulerPoincaré, existe uma transformação contínua $f: P \longrightarrow Q$ cuja inversa $f^{-1}: Q \longrightarrow P$ também é contínua. Nesse caso, a função $f$ é chamada de homeomorfismo e dizemos que os poliedros $P$ e $Q$ são homeomorfos.

A seguir, nos exemplos 2.1 e 2.2, apresentamos um poliedro homeomorfo à esfera e outro homeomorfo ao toro. De maneira intuitiva, se imaginarmos um poliedro regular feito de borracha e o inflarmos, injetando ar (não precisa ser regular, nem mesmo convexo basta que não possua espaços vazios, buracos, como um toro), ele será transformado em uma esfera. Nesse caso seu invariante topológico é $X(P)=V-A+F=2$ (Fórmula de Euler). Já os poliedros que têm um único buraco serão transformados em um toro (câmara de pneu cheia de ar) e seu invariante topológico é $X(P)=V-A+F=0$.

No caso dos exemplos é fácil contar o número de faces, arestas e vértices e concluir o resultado; mais especificamente, os objetos homeomorfos à esfera têm característica 2 e os homeomorfos ao toro característica 0.

Ademais, sabe-se que toda superfície compacta pode ser triangularizável, isto é, pode ser apresentada em forma de poliedro formado por faces triangulares e cujas faces só compartilham arestas e vértices (de forma mais generalizada, podemos cobrir a superfície compacta por um complexo simplicial), mas nesse caso as ferramentas para o calculo da Característica de Euler-Poicaré vão 
se tornando cada vez mais complexas, a ponto de se perderem as noções intuitivas da Geometria Euclideana.

Exemplo 2.1. O poliedro da Figura 1 é homeomorfo à esfera.
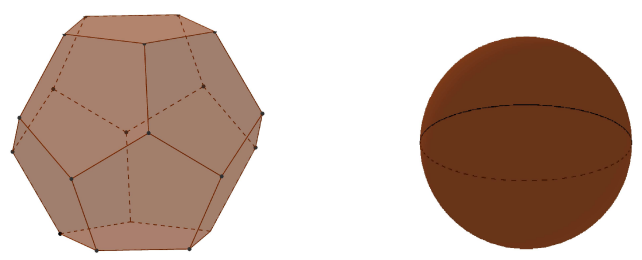

Figura 1: Poliedro homeomorfo à esfera 
Exemplo 2.2. O poliedro da Figura 2 é homeomorfo a um toro.
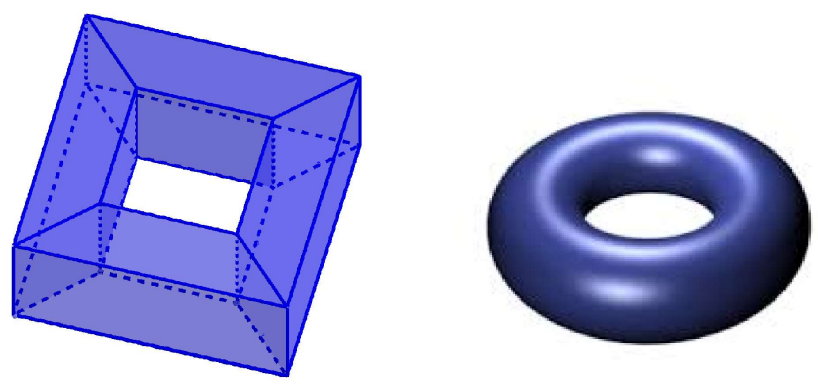

Figura 2: Poliedro homeomorfo a um toro

Igualmente homeomorfa ao toro, a figura a seguir possui a Característica de Euler-Poincaré igual a 0 , porém, é óbvio que, ao contarmos o número de faces, arestas e vértices constatamos que a tarefa não é tão fácil.

É irrelevante a forma poliédrica que se retrate um toro, desde que se tenha a propriedade que as faces só possuam em comum arestas com arestas e vértices com vértices, para que a fórmula $V-A+F$ seja sempre constante e igual a 0 , exatamente por ser esse número um invariante topológico. Tal tarefa, porém, pode se tornar complexa de acordo com a forma escolhida.

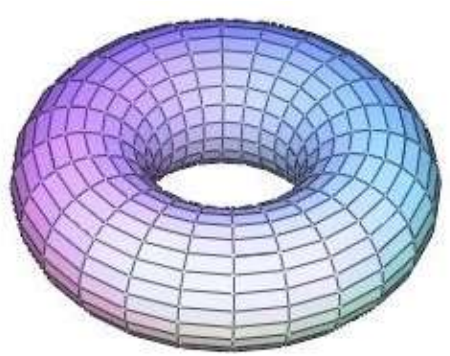

Figura 3: Poliedro homeomorfo a um toro 


\subsection{Poliedros $n$-tóricos}

Nesta seção, tratamos de poliedros com buracos no formato do exemplo 2.2. Percebemos que a Fórmula de Euler não está satisfeita para esse poliedro com buraco, já que $V-A+F=0$ e esperávamos que esse valor fosse 2 , e isso ocorre exatamente porque esse objeto não é homeomorfo à esfera.

Dessa forma, usando apenas indução finita, demonstraremos o valor da Característica de EulerPoincaré para uma classe de poliedros homeomorfos aos $n$-toros.

$\mathrm{Na}$ verdade, o que necessitamos é uma classe de objetos homeomorfos aos $n$-toros que sejam construídos de forma poliédrica, sendo que o número de faces, arestas e vértices, sejam controladas à medida que se adere a ele um novo toro.

Entre as provas da validade da Característica de Euler-Poincaré está a utilização de complexos simpliciais; primeiro mostra-se que toda superfície compacta pode ser triangularizável, depois estuda-se o que ocorre quando se unem simplexos, e, finalmente prova-se a validade do invariante. Para conhecer mais sobre esses resultados temos uma classificação completa das superfícies compactas na dissertação de $\operatorname{SOUZA(2016)}$ ou um estudo mais algébrico utilizando simplexos no livro de $\mathrm{HU}(1966)$ e, ainda, algumas aplicações destes resultados podem ser encontradas no livro de CARMO(2006).

O que faremos é uma simplificação desse processo. Para isso criaremos uma definição intuitiva de uma forma poliédrica de $n$-toros, como nos exemplos a seguir.

Exemplo 2.3. Consideremos um poliedro 1-tórico, conforme Figura 4. A Característica de EulerPoincaré do poliedro 1-tórico é dada por

$$
X(P)=V-A+F=16-32+16=0 .
$$

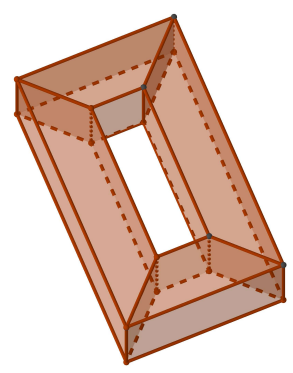

Figura 4: 1-tórico.

Exemplo 2.4. Consideremos um poliedro 2-tórico, veja Figura 5. A Característica de Euler-Poincaré do poliedro 2-tórico é dada por

$$
X(P)=V-A+F=24-48+22=-2 .
$$




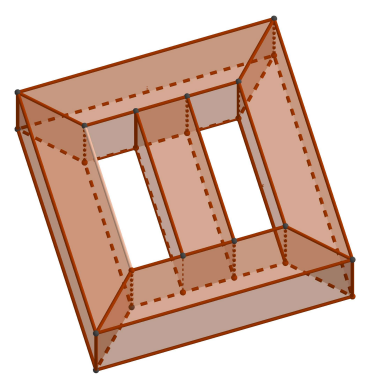

Figura 5: 2-tórico

Exemplo 2.5. Para 3-tórico, temos

$$
X(P)=V-A+F=32-64+28=-4 .
$$

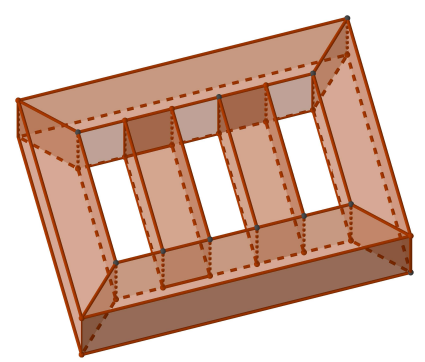

Figura 6: 3-tórico

Definição 2.6 (Poliedros $n$-tóricos). Em linhas gerais, denominamos de poliedros $n$-tóricos os poliedros, retos ou oblíquos cujas faces são paralelogramos e em sua constituição final aparecem com $n$ buracos construídos de forma que as faces internas, que constituem os buracos, são paralelas às faces externas, conforme os exemplos anteriores.

Pretendemos demonstrar, por indução finita, a Característica de Euler-Poincaré para poliedros $n$-tóricos.

Calculando a Característica de Euler-Poincaré dos poliedros das Figuras 4, 5 e 6, obtemos termos de uma progressão aritmética, a saber $(0,-2,-4, \ldots)$, de razão $r=-2$, onde o primeiro termo é $a_{1}=0$. Assim, o termo geral $a_{n}$ dessa progressão é dado por

$$
a_{n}=a_{1}+(n-1) \cdot r=0+(n-1) \cdot(-2)=2-2 n, \quad n \in \mathbb{N} .
$$


Esse resultado motiva-nos a enunciar uma reformulação da conhecida Fórmula de Euler, que corresponderá a Característica de Euler-Poincaré para poliedros n-tóricos.

Teorema 2.7. Seja $P$ um poliedro $n$-tórico com $V$ vértices, $A$ arestas e $F$ faces. Então, a Característica de Euler-Poincaré de $P$ é dada por

$$
X(P)=V-A+F=2-2 n,
$$

onde $n \in \mathbb{N}$ e $n \geq 1$.

Demonstração. A demonstração será feita por indução sob $n \in \mathbb{N}$. Para o 1-tórico, conforme Figura 4, temos

$$
X(P)=V-A+F=16-32+16=0,
$$

satisfazendo (1).

Suponhamos a fórmula (1) válida para um poliedro $n$-tórico, $n>1$.

$$
X(P)=V_{n}-A_{n}+F_{n}=2-2 n,
$$

onde $V_{n}, A_{n}$ e $F_{n}$ denotam o número de vértices, arestas e faces, respectivamente, do poliedro $n$-tórico.

Nosso objetivo é provar que a fórmula (1) vale para poliedros com $n+1$ buracos.

Para concluirmos a prova, vamos examinar o que acontece quando incluímos mais um buraco em poliedro um n-tórico.

Como ilustração vamos unir os poliedros das Figuras 4 e 5 por meio de suas faces (esse procedimento topológico é chamado de cirurgia ou soma conexa).

Notemos que, para o poliedro da Figura 5, vale $X(P)=V_{2}-A_{2}+F_{2}=2-2(2)$. Ao "colarmos" os dois poliedros das Figuras 5 e 4, a saber o 2-tórico e o 1-tórico, alguns vértices, faces e arestas tornar-se-ão apenas um. E obtemos um novo poliedro com um buraco a mais, ou seja, um poliedro 3 -tórico, Figura 6, para o qual podemos contar e verificar que $X(P)=V_{3}-A_{3}+F_{3}=2-2(3)$.

De fato, isso é exatamente o que ocorre no caso geral quando unimos convenientemente um poliedro n-tórico a um poliedro 1-tórico, para obter um poliedro $n+1$-tórico. O que precisamos estudar é o que ocorrem com os vértices, arestas e faces nesse procedimento para obtermos o caso geral. 

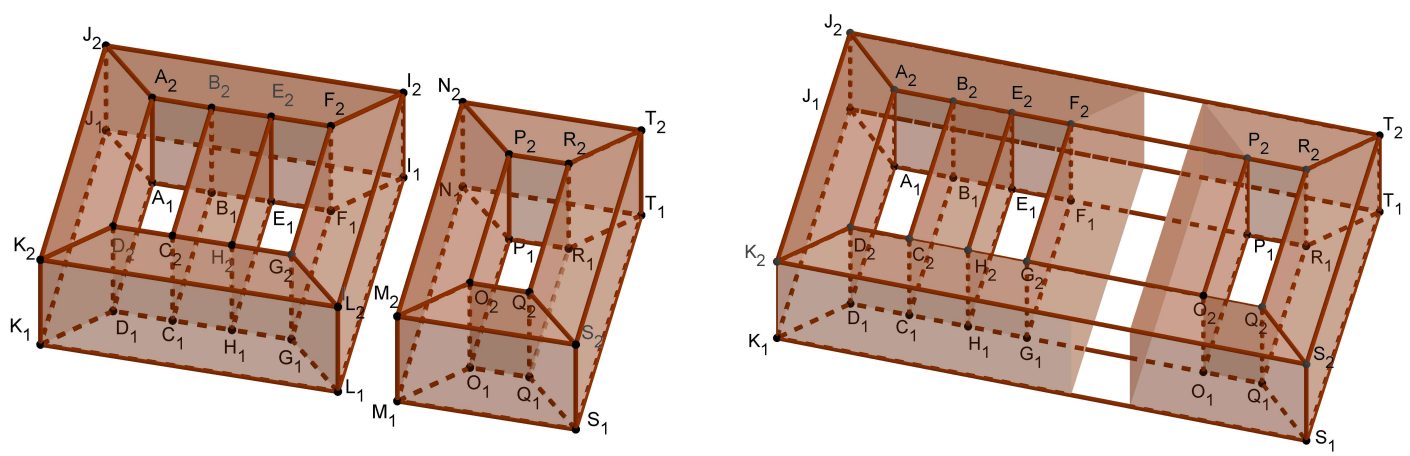

Figura 7: $(2+1)$-tórico

A seguir, apresentamos a demonstração geral de forma detalhada.

Com relação aos vértices $V_{n}$ do poliedro n-tórico, ao juntarmos os poliedros como nas Figuras 7, o novo poliedro terá 16 vértices a mais oriundos do poliedro da Figura 4, porém 4 vértices de cada figura tornar-se-ão apenas um, a saber, $\left(I_{1}, I_{2}, L_{1}, L_{2}\right)$ e $\left(M_{1}, M_{2}, N_{1}, N_{2}\right)$. Desta forma, o número de vértices obtidos em $V_{n+1}$ é dado por $V_{n}+16-8$.

Com relação às arestas, observamos que as $A_{n}$ arestas do poliedro n-tórico serão acrescentadas as 32 arestas da Figura 4, porém muitas arestas acabaram por se unir, a saber,

$$
\begin{gathered}
\left(I_{1} I_{2}, L_{1} L_{2}, I_{1} L_{1}, I_{2} L_{2}\right), \\
\left(M_{1} M_{2}, N_{1} N_{2}, M_{1} N_{1}, M_{2} N_{2}\right), \\
\left(F_{1} I_{1}, F_{2} I_{2}, G_{1} L_{1}, G_{2} L_{2}\right), \\
\left(M_{1} O_{1}, M_{2} O_{2}, N_{1} P_{1}, N_{2} P_{2}\right), \\
\left(I_{1} J_{1}, I_{2} J_{2}, K_{1} L_{1}, K_{2} L_{2}\right), \\
\left(M_{1} S_{1}, M_{2} S_{2}, N_{1} T_{1}, N_{2} T_{2}\right) .
\end{gathered}
$$

Ainda novas arestas serão formadas; veja

$$
\left(K_{1} S_{1}, K_{2} S_{2}, J_{1} T_{1}, J_{2} T_{2}\right),\left(F_{1} P_{1}, F_{2} P_{2}, G_{1} O_{1}, G_{2} O_{2}\right) .
$$

Daí, obtemos o seguinte número de arestas

$$
A_{n+1}=A_{n}+32-24+8 .
$$


Com relação às faces do novo poliedro, temos que as $F_{n}$ faces do poliedro da n-tórico serão acrescentadas às 16 faces do poliedro da Figura 4, porém algumas faces unir-se-ão, a saber,

$$
\begin{gathered}
\left(I_{1} I_{2} L_{1} L_{2}, M_{1} M_{2} N_{1} N_{2}\right), \\
\left(F_{1} G_{1} I_{1} L_{1}, F_{2} G_{2} I_{2} L_{2}, M_{1} N_{1} O_{1} P_{1}, M_{2} N_{2} O_{2} P_{2}\right), \\
\left(D_{1} G_{1} K_{1} L_{1}, D_{2} G_{2} K_{2} L_{2}, A_{1} F_{1} I_{1} J_{1}, A_{2} F_{2} I_{2} J_{2}, M_{1} O_{1} Q_{1} S_{1}, M_{2} O_{2} Q_{2} S_{2}, N_{1} P_{1} R_{1} T_{1}, N_{2} P_{2} R_{2} T_{2}\right)
\end{gathered}
$$

e

$$
\left(K_{1} K_{2} L_{1} L_{2}, M_{1} M_{2} S_{1} S_{2}, I_{1} I_{2} J_{1} J_{2}, N_{1} N_{2} T_{1} T_{2}\right)
$$

dando origem às faces

$$
\begin{gathered}
\left(F_{1} G_{1} O_{1} P_{1}, F_{2} G_{2} O_{2} P_{2}\right), \\
\left(D_{1} K_{1} Q_{1} S_{1}, D_{2} K_{2} Q_{2} S_{2}, A_{1} J_{1} R_{1} T_{1}, A_{2} J_{2} R_{2} T_{2}\right)
\end{gathered}
$$

e

$$
\left(J_{1} J_{2} T_{1} T_{2}, K_{1} K_{2} S_{1} S_{2}\right)
$$

Logo, obtemos o seguinte número de faces do $(n+1)$-tórico, como mostra a Figura 8 ,

$$
F_{n+1}=F_{n}+16-18+8 .
$$

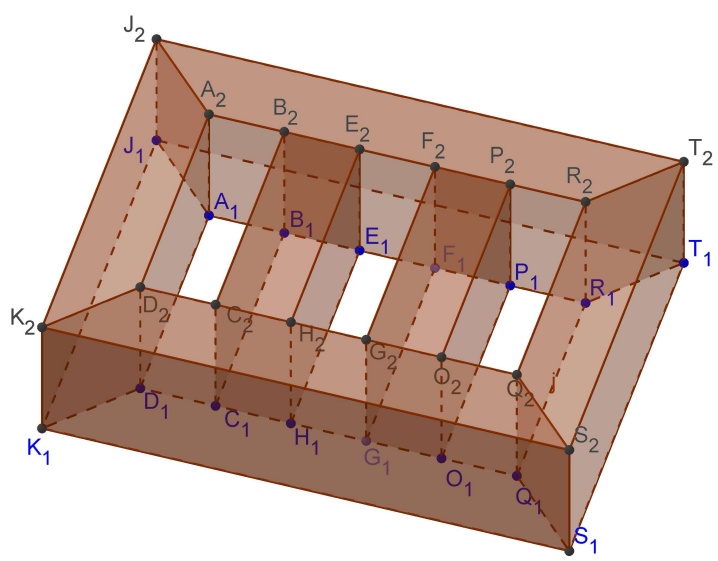

Figura 8: $(n+1)$-tórico

Agora, em particular, para o poliedro $(n+1)$-tórico, $V_{n+1}-A_{n+1}+F_{n+1}=V_{n}+16-8-\left(A_{n}+32-24+8\right)+F_{n}+16-18+8=V_{n}+8-A_{n}-16+F_{n}+6$. 
Logo,

$$
V_{n+1}-A_{n+1}+F_{n+1}=V_{n}-A_{n}+F_{n}-2=2-2 n-2=2-2(n+1) .
$$

Portanto, a fórmula (1) é válida para poliedros com $n+1$ buracos, consequentemente,

$$
X(P)=V-A+F=2-2 n,
$$

é válida para $n \in \mathbb{N}$ e $n \geq 1$, provando o resultado desejado.

\section{Considerações finais}

Os poliedros aqui chamados de $n$-tóricos são versões poliédricas de $n$-toros, para os quais se conhece muito bem a Característica de Euler-Poincaré- entretanto, por se tratar de invariante topológico, em geral, para sua apresentação faz-se necessário um ferramental topológico muito grande, como, os simplexos, as cirurgias ou somas conexas, entre outros.

Como vimos na Figura 3, a escolha da figura poliédrica homeomorfa a um $n$-toro é o que diferencia a capacidade de se contar o número de faces, arestas e vértices. Assim, para simplificarmos a prova do valor da Característica precisamos fazer uma escolha planejada - neste caso os poliedros $n$-tóricos.

Por outro lado, é claro que o presente artigo lança mão das mesmas ferramentas topológicas, como os simplexos, uma vez que faces poliédricas formam um simplexo, mas o faz de maneira menos elaborada - demonstramos esse fato usando a boa e velha observação (tão usada na geometria ao longo da história) e a indução finita.

Tal resultado, assim, passa a ser relevante como ferramenta para melhorar ainda mais as possibilidades de sedução (já mencionadas) da geometria aos jovens estudantes. Não esperamos, portanto, que esse trabalho seja visto como inovador mas como uma forma diferente e, talvez, mais atraente de ver a geometria envolvida neste resultado.

De forma muito similar pode-se, ainda, definir a garrafa de Klein poliédrica (infelizmente não tão intuitiva como os $n$-toros, veja [8],[9]) e verificar que a fórmula ainda é válida para objetos de característica positiva.

\section{Agradecimentos}

Agradecimento especial ao Mestrado Profissional em Matemática em Rede Nacional - Profmat

\section{Referências}

[1] CARMO, M.P. do Geometria Diferencial de Curvas e Superfícies. $2^{a}$ ed. Rio de Janeiro: SBM. 2006.

[2] GISOLDI, D. V. A característica de Euler. 43f. Dissertação (Mestrado Profissional em Matemática), Orientador: Alice Kimie Miwa Libardi. Universidade Estadual Paulista, Rio Claro. 2013.

[3] HU, Sze-Tse. Homology Theory: a first course in algebraic topology. San Francisco: Holden-Day, Inc. 1966. 
[4] LIMA, E. L. Meu Professor de Matemática e Outras Histórias. Rio de Janeiro: SBM. 1991.

[5] Lima, E. L., CARVAlho, P. C. P., MORGAdO, A. C., WAGNER, E., A Matemática do Ensino Médio. vol. 2. Rio de Janeiro: SBM. 1999.

[6] PEDROCHI, W. E. Teorema de Euler para poliedros não convexos. 63f. Dissertação (Mestrado Profissional em Matemática), Orientador: Rodrigo Martins. Universidade Estadual de Maringá, Maringá. 2016.

[7] SOUZA, J. Novo Olhar Matemática. vol. 3. São Paulo: FTD. 2013.

[8] SOUZA, A. F. M. de. Um estudo sobre a classificação topológica das superfícies. 86f. Dissertação (Mestrado em Matemática), Orientadora: Thaís F. M. Monís. Universidade Estadual Paulista, Rio Claro, 2016.

[9] WOLFRAM MathWorld. Klein Bottle. Disponível em: http://mathworld.wolfram.com/KleinBottle.html. Acesso em: 15 de set. 2019.

Luciene Parron Gimenes Arantes Universidade Estadual de Maringá (UEM), DMA, Maringá, PR, Brasil $<$ lpgarantes@uem.br>

Wellen Eder Pedrochi Universidade Estadual de Maringá (UEM), ProfMat, Maringá, PR, Brasil $<$ wpedrochi@hotmail.com>

Rodrigo Martins

Universidade Estadual de Maringá (UEM), DMA, Maringá, PR, Brasil <rmartins@uem.br>

Recebido: $11 / 06 / 2019$

Publicado: 24/10/2019 